\title{
Three-Point Electromagnetic Spider Web
}

\author{
Min Jiang \\ Ningbo Polytechnic, Ningbo, China \\ Email: chiangm@163.com
}

How to cite this paper: Jiang, M. (2017) Three-Point Electromagnetic Spider Web. Open Access Library Journal, 4: e3582. https://doi.org/10.4236/oalib.1103582

Received: April 6, 2017

Accepted: May 13, 2017

Published: May 16, 2017

Copyright $\odot 2017$ by author and Open Access Library Inc.

This work is licensed under the Creative Commons Attribution International License (CC BY 4.0).

http://creativecommons.org/licenses/by/4.0/

\begin{abstract}
Simple three-point electromagnetic spider web combined with the host and the $30 \mathrm{~m}, 300 \mathrm{~m}, 3000 \mathrm{~m} 3$ transmitters and 3 receiver circuits, telluric electricity and magnetic switch. Here the space electric field change by using the designed solenoid spider does not need deep inside the earth, and a seismometer and earthquake prediction control system is simple. After years of testing, with the use of every minute it is four times 5 - 10 second detection magnetic anomaly, and abnormal situation will be asked after the three behind the road before the earthquake electromagnetic parameters are converted to a digital signal electric parameter. If finding electromagnetic anomaly in 30 square kilometers will start the GSM switch remote alarm, using Maxwell's equations theory and practice the test showed that this method can not only be used for electromagnetic anomaly before the earthquake prediction, and a plurality of electromagnetic webs can determine epicenter group composition. Before the earthquake changes in the electromagnetic field in a few hours to a few days, we are able to know in time that an earthquake is about to occur, and determine the location of the epicenter. The devices may be detected over stealth aircraft [1] [2] [3].
\end{abstract}

\section{Subject Areas}

Environmental Sciences

\section{Keywords}

Electromagnetic Spider Web, Telluric Electricity, Mat-Lab Simulate, Earthquake Prediction, Magnetic Switch Module, Wireless Transmitting and Receiving Module, GSM

\section{The Principle of Maxwell's Equations}

Our design is the theoretical basis of Maxwell's equations. Maxwell's equations are to describe the relationship between electric field and magnetic field and 
charge density and current density of the partial differential equations of a group of British physicist James Maxwell established in nineteenth Century. It consists of four equations: describing how to produce electric charge of Gauss's law; discussing magnetic monopoles do not exist; and the description of the current law of Gauss magnetic time varying electric field magnetic field generated by Maxwell to describe the time-varying magnetic field Ampere law; how to produce the Faraday's law of induction electric field. From the Maxwell equations, we can infer that the electromagnetic wave propagates in the vacuum at the speed of light, and then make the assumption that the light is electromagnetic waves. Maxwell's equations and the Lorenz force equation are the basic equations of classical electromagnetics. From the basic theory to these basic equations, the original form of the equations proposed by Maxwell in 1865 consists of 20 equations and 20 variables. He tried to use quaternion in 1873, but did not succeed. The mathematical form now used is Heaviside and Gibbs 1884 to form the new expression vector analysis. Electromagnetic spider web is an actually a large radius of the electromagnetic induction experiment (Figure 1).

\section{Space Electromagnetic Field Sensor Structure}

Production is placed 3 principle of wireless transmission module in earthquake prone areas, placed distance of $30 \mathrm{~m}, 300 \mathrm{~m}, 3000 \mathrm{~m}$ using the corresponding super heterodyne, reflex circuit receiving device receives signals. The electronic compass and telluric electricity each placed in the determination of earth magnetic anomaly remote monitoring based on GPRS network, a plurality of electromagnetic spider webs and electromagnetic group hang together by the GPRS network can judge, a week before the earthquake measured changes in the local electromagnetic field before the earthquake early warning, but also analyzed the quake epicenter. The electromagnetic spider web to make up for deficiencies in the research before, and if multiple electromagnetic cobwebs on the earth, and the regular collection of remote electromagnetic comprehensive analysis, it is convenient to form three-dimensional research, measured three factors of earthquake. Figure 2 is three point electromagnetic spider web diagrams. Electromagnetic spider web is three-dimensional method to detect earthquakes, theoretically more advanced than the point and line. Einstein once said: "Imagination is more important than knowledge". The receiver will use mathematical

$$
\left\{\begin{array} { l l } 
{ \oint _ { S } \vec { D } \cdot d \vec { S } = \int _ { V } \rho d V } \\
{ \oint _ { S } \vec { B } \cdot d \vec { S } = 0 } \\
{ \oint _ { L } \vec { E } \cdot d \vec { l } = - \frac { d \Phi _ { m } } { d t } } \\
{ \oint _ { L } \vec { H } \cdot d \vec { l } = \sum I + \frac { d \Phi _ { D } } { d t } }
\end{array} \quad \left\{\begin{array}{l}
\nabla \cdot \vec{D}=\rho \\
\nabla \cdot \vec{B}=0 \\
\nabla \times \vec{E}=-\frac{\partial \vec{B}}{\partial t} \\
\nabla \times \vec{H}=\vec{j}+\frac{\partial \vec{D}}{\partial t}
\end{array}\right.\right.
$$

Figure 1. Schematic diagram of the Maxwell equation. 

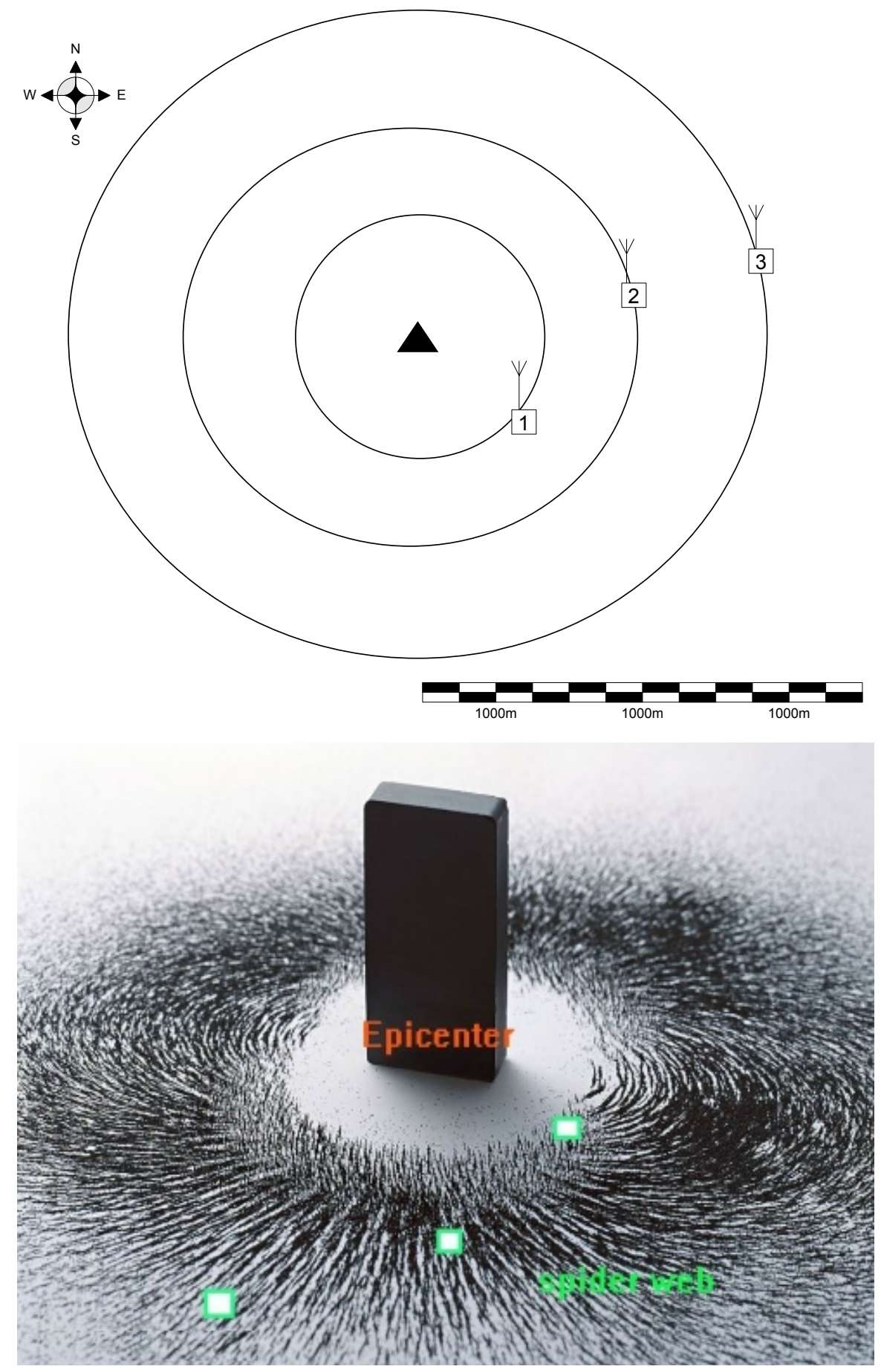

Figure 2. Three point electromagnetic spider web diagram.

statistical analysis of Mat-lab multi-channel signal, the sensor should be used with no predecessors' invention, and heaven should pay off, if the earthquake prediction can be a breakthrough in science, the reasonable physical interpretation of seismic electromagnetic wave in theory.

In practice, a convenient and practical electric field sensor is developed, which has the ability to surpass the nature in the fight against natural disasters. The electromagnetic spider web, which has been made and placed at a distance, is 
made up of three channels and a three receiver circuit control and test, judge and use. Based on the principle of advanced electrodynamics, the seismic dynamic equation established by Maxwell equation, which is based on the principle of advanced electrodynamics is a little complicated. To know the location of the epicenter of the earthquake, it is necessary to build multiple electromagnetic spider webs [4] [5] [6].

Telluric currents are phenomena observed in the Earth's crust and mantle. In September 1862, an experiment to specifically address Earth currents was carried out in the Munich Alps (Lamont, 1862). Including minor processes, there are at least thirty-two different mechanisms which cause telluric currents. The strongest are primarily geomagnetic ally induced currents, which are induced by changes in the outer part of the Earth's magnetic field, which are usually caused by interactions between the solar wind and the magnetosphere or solar radiation effects on the ionosphere. Telluric currents flow in the surface layers of the earth. The electric potential on the Earth's surface can be measured at different points, enabling the calculation of the magnitudes and directions of the telluric currents and hence the Earth's conductance. These currents are known to have diurnal characteristics wherein the general direction of flow is towards the sun. Telluric currents continuously move between the sunlit and shadowed sides of the earth, toward the equator on the side of the earth facing the sun (that is, during the day), and toward the poles on the night side of the planet. Both telluric and magneto telluric methods are used for exploring the structure beneath the Earth's surface (such as in industrial prospecting). For mineral exploration the targets are any subsurface structure with a distinguishable resistance in comparison to its surroundings. Uses include geothermal exploration, mining exploration, petroleum exploration, mapping of fault zones, ground water exploration and monitoring, investigation of magma chambers, and investigation of boundaries of tectonic plates. Earth batteries tap a useful low voltage current from telluric currents, and were used for telegraph systems as far back as the 1840s. In industrial prospecting activity that uses the telluric current method, electrodes are properly located on the ground to sense the voltage difference between locations caused by the oscillatory telluric currents. It is recognized that a low frequency window (LFW) exists when telluric currents pass through the earth's substrata. In the frequencies of the LFW, the earth acts as a conductor.

When the plane flew over the network of electromagnetic spiders web on equipment, land power device generally have a significant change in $10 \mu \mathrm{A}-20$ $\mu \mathrm{A}$. Ordinary aircraft on the ground is generally difficult to eliminate the impact of the electromagnetic spider web can make flying over the prototype exposure. Figure 3 is simple telluric electricity.

Because the earth is a complex giant system, it is impossible to establish a very accurate kinetic equation. Here the space electric field change by the design and design of network spider does not need a solenoid deep within the earth, a seismometer and earthquake prediction control system is simple. Preliminary experiments have reached the basic design requirements. The electric field and 


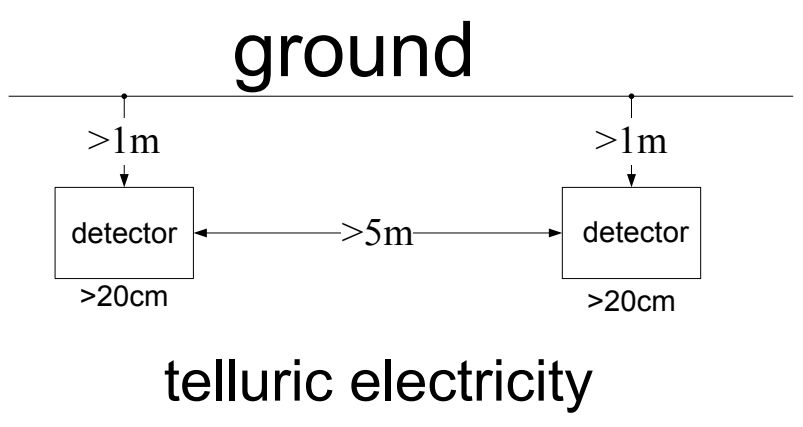

Figure 3. Simple telluric electricity.

magnetic field will be distributed in some form. Figure 4 is the electromagnetic spider and single chip circuit schematic. Simply said: the principle is to receive electromagnetic field using the electromagnetic web production method is before the earthquake, a number of radio signal receiving exception can be conveniently at the receiving end to detect abnormal signal by the number 0,1 way output. The utility model has the advantages of convenient use, low cost and wide use [7] [8] [9].

\section{Remote GSM Network Alarm System}

The equipment with low cost and low power consumption in outdoor equipment is a promising method for short and imminent earthquake prediction. Use $(30 \mathrm{~m}, 300 \mathrm{~m}, 3000 \mathrm{~m})$ distance wireless remote control transmitter, by three road three road combined receiver circuit which consists of electromagnetic cobweb in the range of 30 square kilometers of induction to electromagnetic anomaly ranges light changes the main principle of early warning device making use of electromagnetic spider web is usually in the micro power standby device, each hours of transmitting and receiving electromagnetic field with a variety of bands, that receives the remote signal or not receiving the close signal automatic remote alarm, multiple web connected groups once electromagnetic detection, using the configuration software can be easily remote detective epicenter. Here the electromagnetic spider combined GSM network or GPRS network remote control is composed of three road, three road emission receiving circuit, using multiple relay circuit can make the detection distance is greatly increased, the composition of the network spider electromagnetic and computer networking, by specialized software for judgment can be determined accurately the location of the earthquake epicenter. The principle of network spider electromagnetic is self-multiple transmitter and receiver using computer software to determine the electromagnetic anomaly range, determine the type of earthquake, epicenter and alarm. The simplest GPRS control we chose the second generation of GSM remote control here GSM (Global System for Mobile Communications). The selfmade equipment has low cost and high reliability, and it can be used to detect the change of the real time electromagnetic field in the way of testing once per ten minute. When the abnormal electromagnetic field appears, you can shorten 


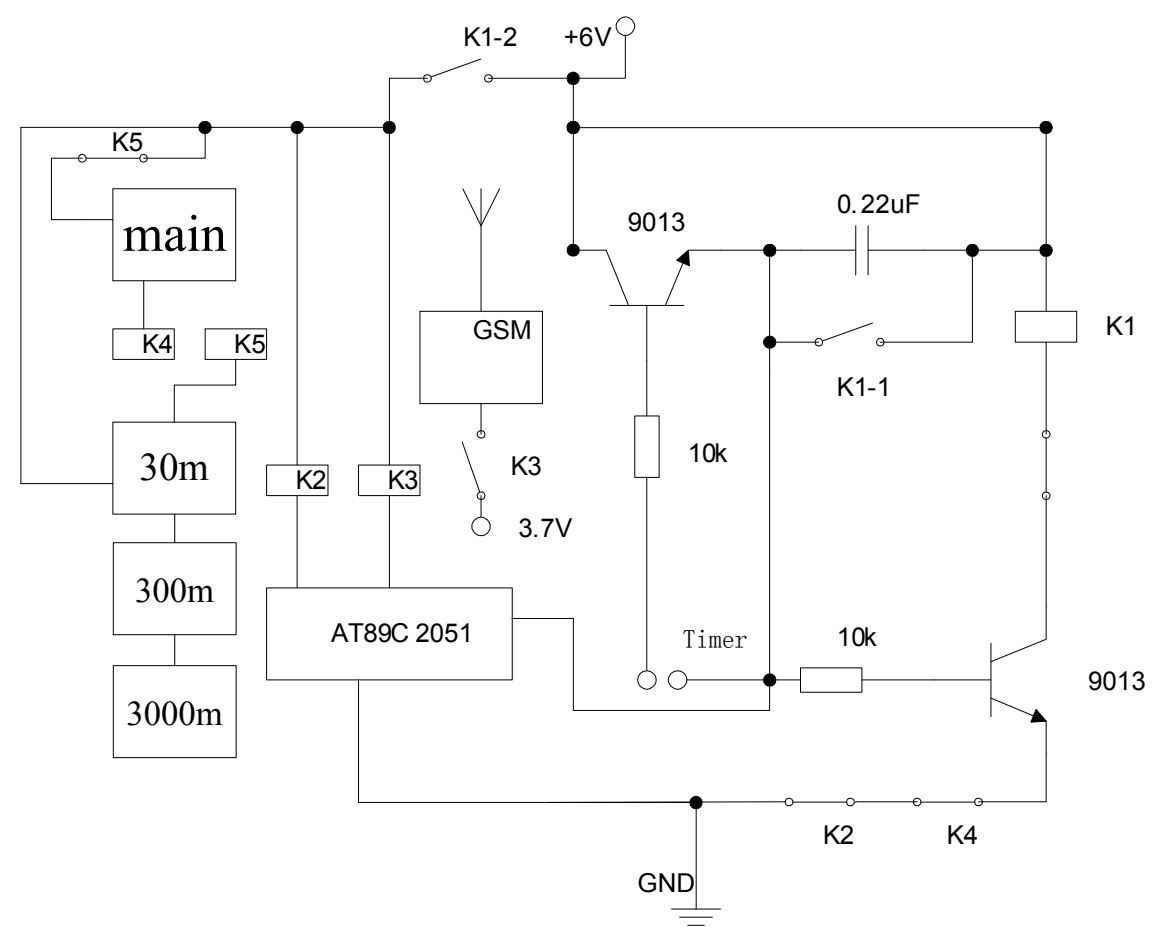

Figure 4. Electromagnetic spider and single chip circuit schematic.

the time, the shortest one minute answer. Globally can available. No distance restriction. With a mobile communication device can control the GPRS finally realize remote wireless alarm. You can simply use the GSM control, can avoid the cumbersome wiring, we can see that the real-time field at home electromagnetic cobwebs in the provinces of cloth, simply plug the SIM card, can work, electricity is easy to use, reliable work. Field placement circuit can be used for more than one year. A series of electrodynamics equations composed of Maxwell's equations are the theoretical basis of the design and the basis of the transmitter. The electronic clock is the core of the design of long time timing transmitting circuit and receiving system. The actual installation requires only three groups.

Remote alarm circuit does not start the GSM in normal electromagnetic, abnormal electromagnetic generated on the remote automatic alarm, alarm selflocking after the release of the remote control to launch the "output off" command. A charge can alarm more than 200 times, very convenient. We design electromagnetic spider webs per hour scan six times, each time is 3 minutes respectively receive the signal received at the receiving end are remote, if multiple electromagnetic spider web groups, combined with the GPRS remote monitoring device can be analyzed accurately in time and location of the earthquake epicenter of the earthquake level. Use the ancient Chinese ten tactics and abnormal electromagnetic field duel to determine the epicenter location. Most of the earthquake damage is great in China. This device can be a few days ahead of time to know a few hours before the earthquake electromagnetic anomalies can avoid many terrible disasters. Has been successfully tried a number of sets, in the 
future there will be a large number of devices placed throughout the country. Four 5\#batteries, the 2 section of the 3.7 volt rechargeable battery life of more than 1 year, generally do not need manual intervention. After the lifting of the remote control to launch the "output off" command, abnormal electromagnetic production on the remote automatic alarm self-locking. Commonly used instructions have "point off", "reply to open", "back off", "output", "output off", "point to open", "point off", "memory" and so on, convenient and practical. At the same time, the low power consumption magnetic field offset detector is composed of an electronic compass and an ordinary compass. Use special timing device to open four times per minute, each time $5-10$ seconds. Before the earthquake, the magneto electric effect is the main factor caused by the earthquake. The impending earthquake prediction for space electric field and the magnetic field of the earth changes, the electric and magnetic parameters measurement is not necessary to enter the interior of the earth, easy realization of technology a major advantage of low power consumption long time offset detector is the magnetic field of the earth can work normally without debugging. Due to the static power consumption of the circuit is only $0.1-0.2 \mu \mathrm{A}$ is very useful to save electricity. Limited to the current level of science and technology, it is impossible to $100 \%$ accurately predict the magnitude of the earthquake time. However, the study of some abnormal phenomena can greatly reduce the loss caused by the earthquake, especially the loss of human life. The advantage of low power consumption long time offset detector design of earth magnetic field variations of the geomagnetic field parameters before the earthquake, when abnormal remote alarm remote start GSM, abnormal electromagnetic, alarm selflocking discharged by remote control to launch the "output" instruction. This practical use of measurement does not have to enter the earth interior, is technically easy to implement, and suitable for large-scale low-cost distribution points. Very suitable placed for the China's vast rural areas. The circuit is switched on once per minute for about $5-10$ seconds four times. Before the earthquake find magnetic field changes immediate alarm. The circuit design scheme is proposed, which is simple and practical, and it is hoped to protect human life and property in earthquake prediction. It can be predicted that can successfully find anomalies of electromagnetic field and electromagnetic wave alarm method, and then form a comprehensive global monitoring system, will promote the emergence and development of earthquake prediction before the earthquake for scientific research and earthquake prediction.

\section{Summary}

A poem summarizes the working principle of this circuit: Electromagnetic spider webs are not esoteric; Maxwell's equations need to learn well. Logarithmic spiral distance is used to measure the gradient. Multi-point placed (telluric electricity) as the temperature (gradient) display. The spider web woven speed compared to the rotation, being careful not to fear the magnetic field. Divergence occurs when the earth's plates move. Change of electromagnetic field before the earthquake should 
be reported quickly. At present, there are a number of top scientists in the world who are studying the use of electromagnetic change prediction before the earthquake. In the earthquake prone areas, we design the electromagnetic spider network to protect the national property that has practical significance. Simple three-point electromagnetic spider web consisted in the host and the $30 \mathrm{~m}, 300 \mathrm{~m}$, $3000 \mathrm{~m} 3$ transmitters and 3 receiver circuits. Here the space electric field change by using the designed solenoid spider does not need deep inside the earth network, and a seismometer and earthquake prediction control system is simple. The devices may be detected over stealth aircraft. Germany has a nursery rhyme that is the effect of a large number of stars in the sky, but God does not count after the statistics will not be less than one. The same is true of abnormal signals before earthquakes. This paper puts forward the design scheme of the three-point electromagnetic spider web, which is simple and practical, and it is hoped to protect human life and property in earthquake prediction [10] [11] [12] [13].

\section{References}

[1] Jiang, M. (1992) Multi Point Timing Control Circuit. Electric World, No. 4, 42.

[2] Jiang, M. (1996) Sound and Light Indicator of Electronic Compass. Electric World, No. 2, 33.

[3] Jiang, M. (2010) Earthquake Prediction Micro Integrated Measurement Recorder. Technology Innovation Herald, No. 29, 22-23.

[4] Jiang, M. (2013) How to Weave the Electromagnetic Spider Web to Predict Earthquakes. 2013 3rd International Conference on Education and Education Management (EEM 2013), Singapore, 15 November 2013, 546-551.

[5] Jiang, M. (2014) Electromagnetic Spider Web Application in Earthquake Prediction. International Core Journal of Scientific Research \& Engineering Index, 111-114.

[6] Jiang, M. (2014) The Group of Electromagnetic Spider Web, Economic. Business Management and Education Innovation (EBMEI 2014), Singapore, 5-6 November 2014, 287-291.

[7] Jiang, M. (2014) Low Power Consumption Electromagnetic Spider Web. 2014 th International Conference on Education and Education Management (EEM 2014), Singapore, 8-9 December 2014, 74-78.

[8] Jiang, M. and Wang, P.Y. (2015) Simple Space Electric Field and Earth Magnetic Anomaly Remote Alarm System. 2015 International Conference on Frontiers of Manufacturing Science and Measuring Technology, 452-458.

[9] Jiang, M. (2016) Application of 3 Kinds of Practical Electromagnetic Spiders in Electromagnetic Spider Web. ICEICE 2016 02083. MATEC Web of Conferences 44.

[10] Jiang, M. (2016) Easily Magnetic Anomalies Earthquake Prediction. MMME 0509 10. https://doi.org/10.1051/matecconf/20166301020

[11] Jiang, M. (2017) Electromagnetic Spider Web Placement in the Longmen Mountain Fault Zone. http://file.scirp.org/pdf/OALibJ_2017031416270208.pdf https://doi.org/10.4236/oalib.1103463

[12] Jiang, M. (2017) Electromagnetic Spider Web Placement in the Southeast Coastal Seismic Belt in China. http://file.scirp.org/pdf/OALibJ_2017020814005426.pdf

[13] Jiang, M. (2017) Simple Electromagnetic Spider Web Placement and Use Method. 2016 International Conference on Engineering and Advanced Technology, Hong Kong, 22-23 December 2016. https://doi.org/10.2991/iceat-16.2017.18 
Submit or recommend next manuscript to OALib Journal and we will provide best service for you:

- Publication frequency: Monthly

- 9 subject areas of science, technology and medicine

- Fair and rigorous peer-review system

- Fast publication process

- Article promotion in various social networking sites (LinkedIn, Facebook, Twitter, etc.)

- Maximum dissemination of your research work

Submit Your Paper Online: Click Here to Submit

Or Contact service@oalib.com 